\title{
Sub-gram in-plane vibration-driven robot with inclined legs
}

\author{
David Robles-Cuenca ${ }^{1 *}$, Victor Ruiz-Díez ${ }^{1}$, José Luis Sánchez-Rojas ${ }^{1}$ and Jorge Hernando-

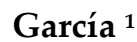

1 Microsystems, Actuators and Sensors Group, Universidad de Castilla-La Mancha, E-13071 Ciudad Real, Spain; david.robles2@alu.uclm.es (D.R-C); victor.ruiz@uclm.es (V.R.-D); joseluis.saldavero@uclm.es (J.L.S.-R.); jorge.hernando@uclm.es (J.H.-G.)

* Correspondence: david.robles2@alu.uclm.es; Tel.:+34-926-295-300

\begin{abstract}
This document reports the design, fabrication and performance of miniaturized locomotion systems employing flexible materials and 3D printed legs. The movement of the system was achieved by the first extensional mode of vibration of the platform of the robot and the inclination of the supporting legs. The structures were manufactured using a $30 \mathrm{~mm}$ long piezoelectric polyvinylidene fluoride (PVDF) film as robot platform, with manually added legs fabricated by stereolithography (SLA). Several speed measurements were performed for samples of $1 \mathrm{~mm}$ and $2 \mathrm{~mm}$ long legs, at an angle of inclination of $45^{\circ}$ and $60^{\circ}$ to the PVDF film. The system was able to exceed a speed of $1 \mathrm{BL} / \mathrm{s}$ to $25 \mathrm{~V}$.
\end{abstract}

Keywords: extensional mode; miniaturized locomotion; piezoelectric; PVDF

\section{Introduction}

Despite the breakthroughs in the locomotion of robots at the macroscale, there is not a counterpart at the miniature scale. A recent review [1] pointed out the limitations of sub-gram systems. Locomotion based on legs is already well established for robotic platforms and might be a solution for miniaturization. Assuming a back and forth motion of the legs, a net displacement can be attained when the forward slip is not equal to the backward slip.

The motion of the legs can be induced by the generation of waves on the platform where the legs are attached. Waves might be either standing waves (SW) or travelling waves (TW). The combination of straight legs and SW was reported for the movement of sliders [1] or the locomotion of a platform [2]. Recent publications also demonstrated the use of TW and straight legs for conveyance applications [3], as well as robotic locomotion [4].

The use of inclined legs is also a common way of achieving an asymmetric slip. Numerous publications implemented this approach [5-7]. In most of these publications a vertical displacement of the robot platform was required to induce the asymmetry and achieve a net horizontal motion.

Here we also propose the use of inclined legs, but with a horizontal displacement of the platform. For that, a SW approach is selected: the generation of the first extensional mode of vibration of a free rectangular platform. The material of the platform is piezoelectric polyvinylidene fluoride (PVDF). This polymer allows for the actuation, as well as for a low cost, light weight approach in the quest of 
miniaturization. Preliminary results demonstrated a locomotion speed of $42 \mathrm{~mm} / \mathrm{s}$ for a $15.8 \mathrm{mg}$ PVDF/legs combination.

\section{Device design}

The core of our design relies on how to combine the extensional mode of vibration of the platform and the inclined legs.

The modal shape of this mode is calculated using equation (1), where $l$ is the maximum displacement, $x$ is the position along the sample with $\mathrm{x}=0$ at one of the edges of the sample and $\mathrm{L}$ is the length of the sample. As it can be seen in figure 1, each side stretches in one direction, reaching its maximum at the edges. Therefore, the legs will be located as close to the edges as possible.

$$
u=l \cos (\pi x / L)
$$

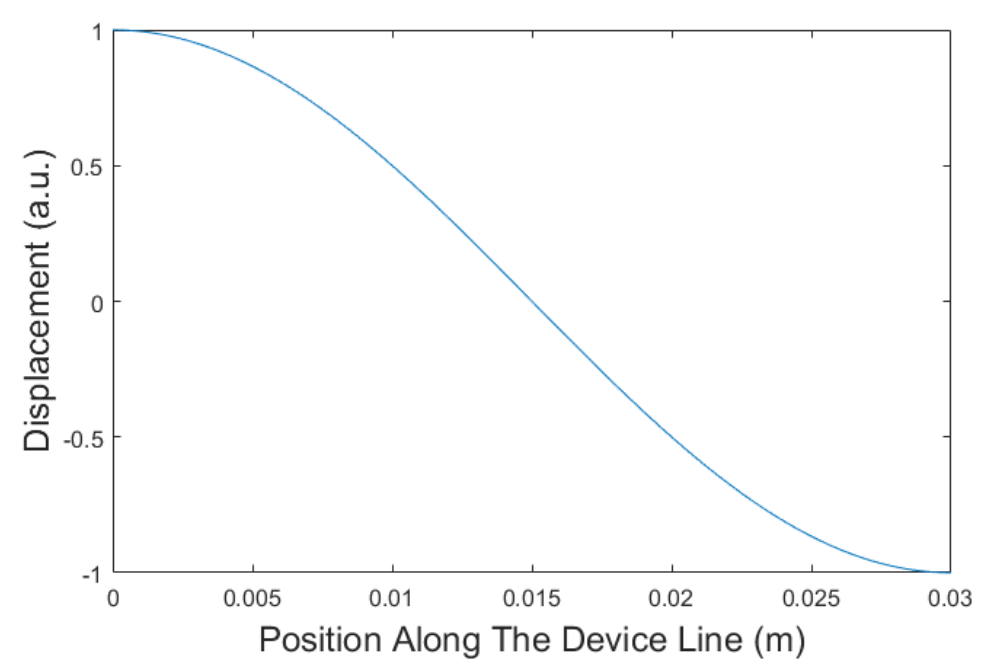

Figure 1. Displacement along the sample due to the extensional mode.

Figure 2 shows the PVDF platform together with the legs. Both the orientation and the location of the legs are important parameters to consider. With the orientation of the figure, the inclined legs show a higher friction when moving to the left than to the right [8]. Besides, due to their location, each leg moves in opposing direction, what results in a net movement of the platform to the right.

The frequency of vibration of the extensional mode was obtained by equation (2) for a free-free beam, as indicated by the reference [9].

$$
f_{0}=\frac{1}{2 L} \sqrt{\frac{E}{\rho}}
$$

Where $\rho$ is the density of the material and E is Young's module of the beam.

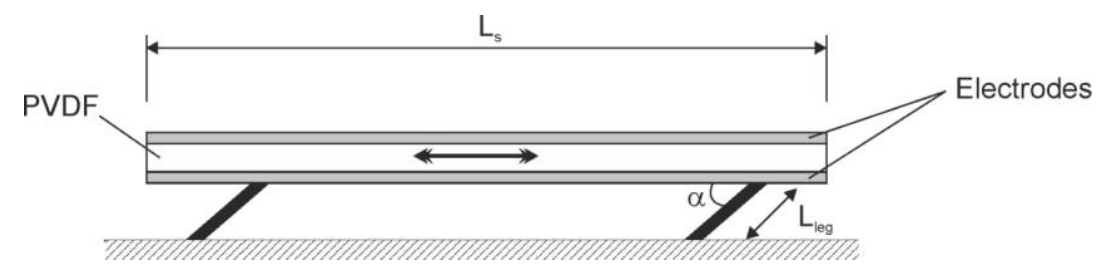

Figure 2. Schematic diagram of the device design. The device consists in a PVDF piezoelectric film metalized at both sides. Two pairs of 3D-printed legs were manually attached to the bottom side of the PVDF film. 


\section{Materials and methods}

The platform of the robot was a $30 \mu \mathrm{m}$ thick uniaxially oriented piezoelectric PVDF layer, metalized on both sides with $\mathrm{Cu} / \mathrm{Ni}$, commercially available from Goodfellow. A die cut machine (Silver Bullet) was used to define the size of the robot: $30 \mathrm{~mm}$ long and $5 \mathrm{~mm}$ wide. With these dimensions, the theoretical frequency of the extensional mode was $17.7 \mathrm{kHz}$, according to equation (2). Table 1 shows the PVDF properties used for the calculation.

Table 1. Properties used in the analysis.

\begin{tabular}{|l|c|l|l|}
\hline Parameter & Symbol & Value & Unit \\
\hline PVDF density $\left(\mathrm{kg} / \mathrm{m}^{3}\right)$ & $\rho$ & 1760 & $\mathrm{~kg} / \mathrm{m}^{3}$ \\
\hline PVDF Young's modulus (GPa) & $E$ & 2 & $\mathrm{GPa}$ \\
\hline
\end{tabular}

Figure 3 shows a picture of the fabricated U-shaped legs. A stereolithography (SLA) 3D printer (B9 Core 530) was used for the fabrication. The material was Nylon resin. The length and the angle of inclination of the legs were varied. $1 \mathrm{~mm}$ and $2 \mathrm{~mm}$ long legs were fabricated, at two different angles of inclination to the PVDF platform, $45^{\circ}$ and $60^{\circ}$. The legs were attached to the platform by means of epoxy resin, an elastic adhesive that prevented stiffening the joint. For electrical access to the metallizations of the PVDF layer, $25 \mu \mathrm{m}$ thick aluminium wire was used, to minimize loading effects. The connection between the aluminium wire and the PVDF metallizations was made by electric paint (Bare Conductive), coated with a layer of silver paint to improve conductance. Figure 4 shows a picture of the whole system, PVDF plus legs and electrical connections.

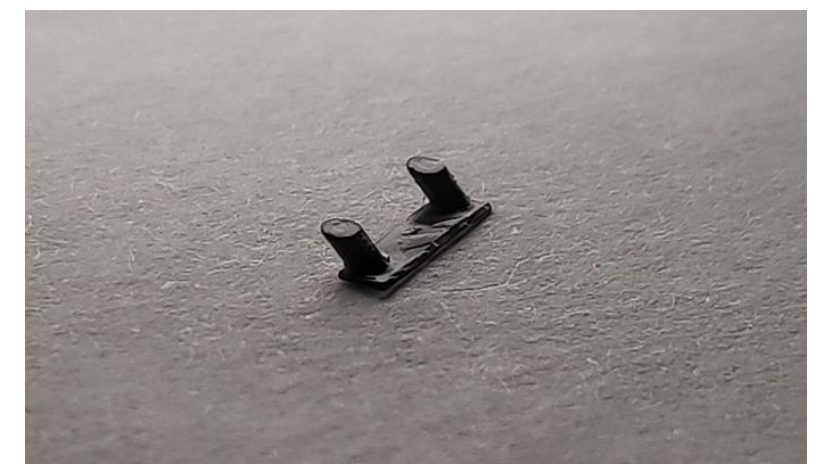

Figure 3. Photograph of one of the U-shaped legs.

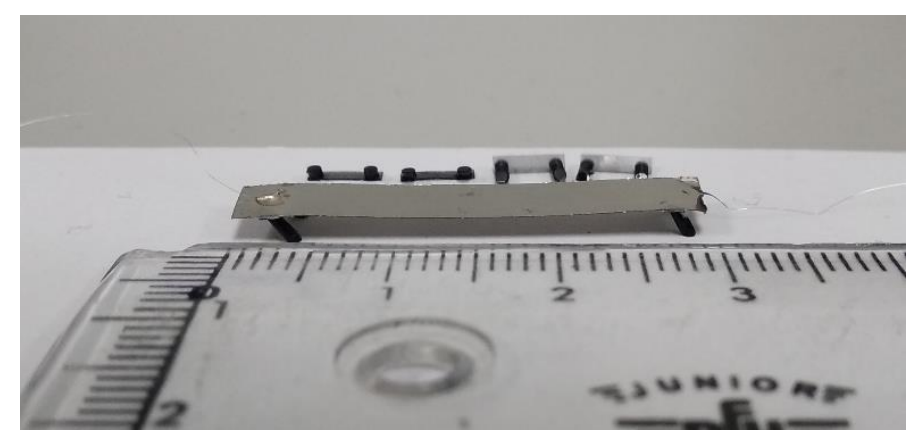

Figure 4. Photograph of the whole system. 
The presence of the extensional mode was detected with the help of an impedance analyser (4294A Agilent). And for the speed characterization of the robot, a set up was mounted with two infrared LEDs, separated $10 \mathrm{~cm}$ and connected to a frequency counter, to detect the pass of the robot.

\section{Results}

Figure 5 shows the electrical conductance of a sample with $2 \mathrm{~mm}$ long legs at an angle of $45^{\circ}$. The maximum response corresponding to the extensional mode was located at about $15.5 \mathrm{kHz}$. This would be the frequency of actuation of the robot. For all the samples under study, the frequency of the maximum conductance of the extensional mode was in the range between 15 and $17 \mathrm{kHz}$. The difference between the theoretical frequency and the measurements might be related to the mass added by the legs and manufacturing defects.

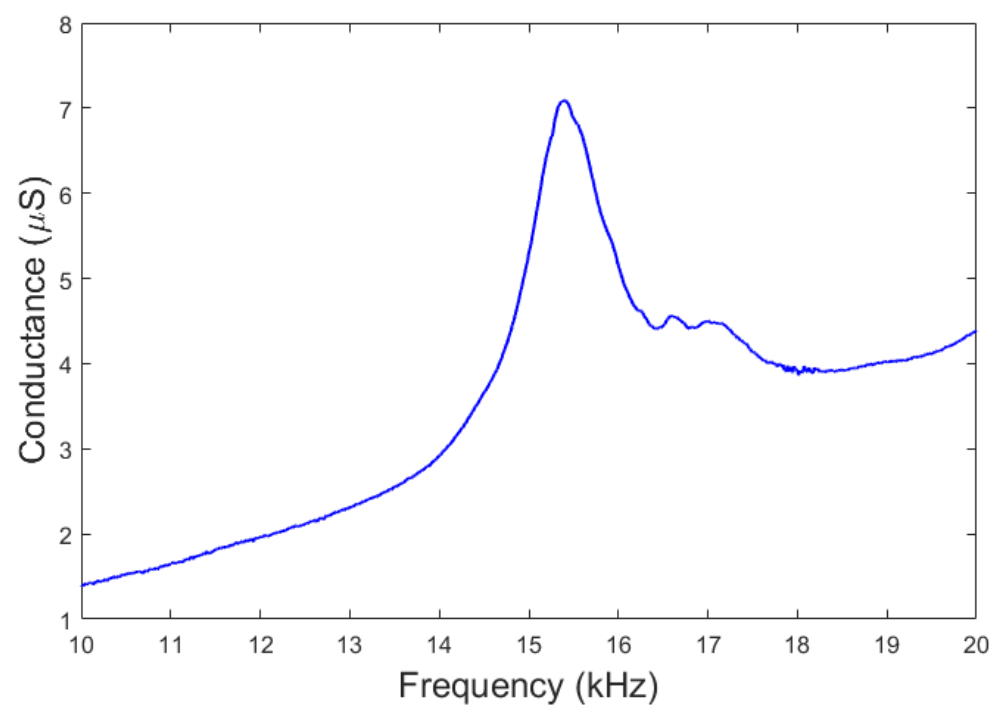

Figure 5. Measured conductance of a sample with a $2 \mathrm{~mm}$ long leg at an angle of $45^{\circ}$

Figure 6 shows the speed of the different samples versus applied voltage. No results were obtained for the robot with $1 \mathrm{~mm}$ long legs at an angle of $45^{\circ}$, mainly due to contact problems between the legs and the floor. A speed as high as $40 \mathrm{~mm} / \mathrm{s}$ was obtained for a voltage of $25 \mathrm{~V}$.

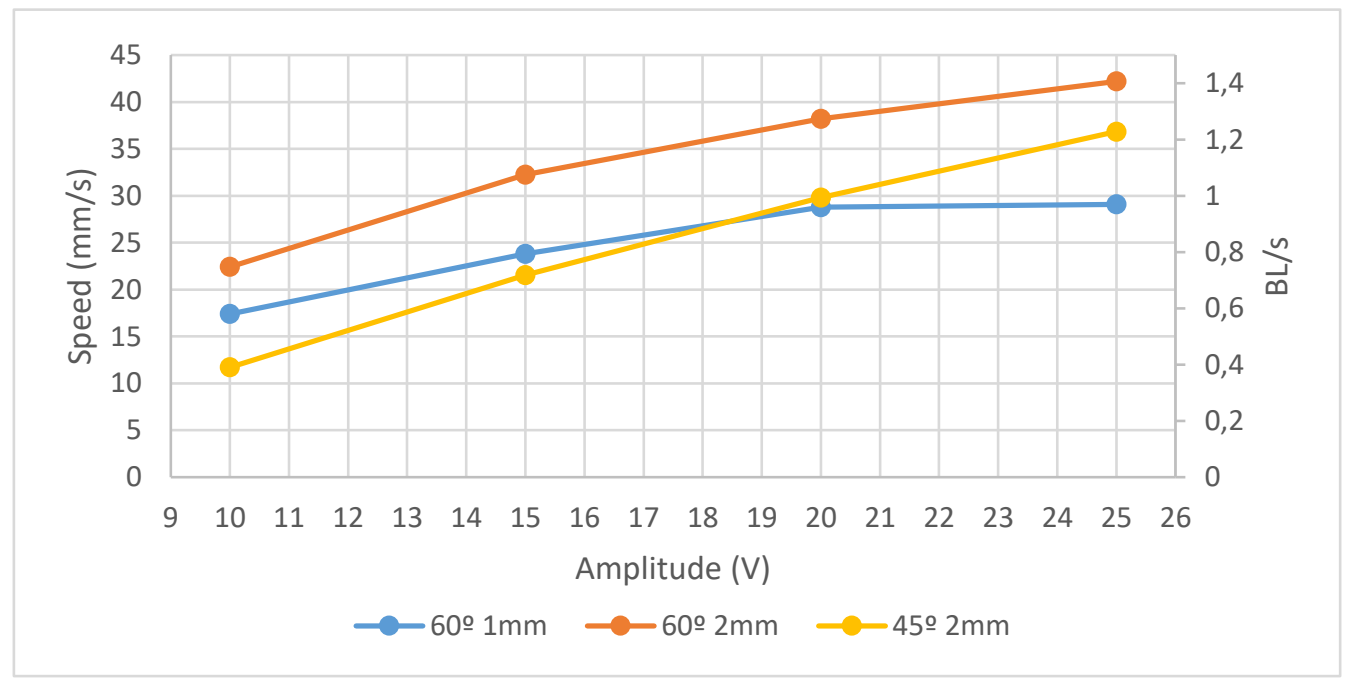

Figure 6. Results from the kinetic characterization of the samples. 


\section{Conclusions}

This work demonstrates the achievement of locomotion by the combination of an extensional mode of vibration and inclined legs. The use of flexible materials resulted in a sub-gram system with a speed of about $1 \mathrm{BL} / \mathrm{s}$ at a voltage of $25 \mathrm{~V}$.

Acknowledgments: This work was supported by the European Regional Development Fund, the Spanish Ministerio de Ciencia, Innovación y Tecnología project (RTI2018-094960-B-100), and the regional government (JCCLM) project (SBPLY/17/180501/000139).

Author Contributions: J.H-G. and J.L.S-R.. conceived and designed the experiments; D.R-C. and V.R-D. performed the experiments and analyzed the data; D.R-C. and J.H-G. wrote the paper.

Conflicts of Interest: The authors declare no conflict of interest. The founding sponsors had no role in the design of the study; in the collection, analyses, or interpretation of data; in the writing of the manuscript, and in the decision to publish the results.

\section{Abbreviations}

The following abbreviations are used in this manuscript:

PVDF: Polyvinylidene fluoride

SLA: Stereolithography

SW: Standing waves

TW: Travelling waves

\section{References}

1. Siyuan He; Weishan Chen; Xie Tao; Zaili Chen Standing wave bi-directional linearly moving ultrasonic motor. IEEE Trans. Ultrason. Ferroelectr. Freq. Control 1998, 45, 1133-1139, doi:10.1109/58.726435.

2. Son, K.J.; Kartik, V.; Wickert, J.A.; Sitti, M. An Ultrasonic Standing-wave-actuated Nano-positioning Walking Robot: Piezoelectric-metal Composite Beam Modeling: J. Vib. Control 2016, doi:10.1177/1077546306070619.

3. Ruiz-Díez, V.; Hernando-García, J.; Toledo, J.; Ababneh, A.; Seidel, H.; Sánchez-Rojas, J.L. Bidirectional Linear Motion by Travelling Waves on Legged Piezoelectric Microfabricated Plates. Micromachines 2020, 11 , 517, doi:10.3390/mi11050517.

4. Hernando-García, J.; García-Caraballo, J.L.; Ruiz-Díez, V.; Sánchez-Rojas, J.L. Motion of a Legged Bidirectional Miniature Piezoelectric Robot Based on Traveling Wave Generation. Micromachines 2020, 11, 321, doi:10.3390/mi11030321.

5. Aoshima, S.; Tsujimura, T.; Yabuta, T. A Miniature Mobile Robot Using Piezo Vibration for Mobility in a Thin Tube. J. Dyn. Syst. Meas. Control 1993, 115, 270-278, doi:10.1115/1.2899031.

6. Giomi, L.; Hawley-Weld, N.; Mahadevan, L. Swarming, swirling and stasis in sequestered bristle-bots. Proc. R. Soc. Math. Phys. Eng. Sci. 2013, 469, 20120637, doi:10.1098/rspa.2012.0637.

7. Shen, Z.; Liu, Y.; Zhao, J.; Tang, X.; Chen, W. Design and Experiment of a Small Legged Robot Operated by the Resonant Vibrations of Cantilever Beams. IEEE Access 2017, 5, 8451-8458, doi:10.1109/ACCESS.2017.2702598.

8. Becker, F.; Börner, S.; Lysenko, V.; Zeidis, I.; Zimmermann, K. On the Mechanics of Bristle-Bots - Modeling, Simulation and Ex- periments. 2014, 6.

9. Blevins, R.D. Formulas for Natural Frequency and Mode Shape; Krieger Publishing Company, 1979; p. 429; ISBN 978-1-57524-184-5. 
() 2020 by the authors; licensee MDPI, Basel, Switzerland. This article is an open access article distributed under (cC) (i) the terms and conditions of the Creative Commons by Attribution (CC-BY) license (http://creativecommons.org/licenses/by/4.0/). 